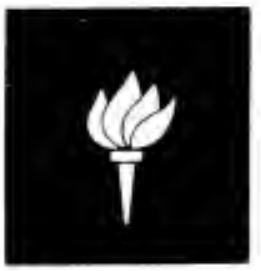

NEW YORK UNIVERSITY

Courant Institute of Mathematical Sciences

Division of Electromagnetic Research

RESEARCH REPORT NO. EMP-207

\title{
ASYMPTOTIC EXPANSIONS OF SOLUTIONS OF INITIAL-BOUNDARY VALUE PROBLEMS FOR A DISPERSIVE HYPERBOLIC EQUATION
}

NORMAN BLEISTEIN

ROBERT M. LEWIS

CONTRACT NO. AF $19(628) 4065$

PROJECT MO.4603

TASK NO.460302

JUNE, 1965

Prepared for

AIR FORCE CAMBRIDGE RESEARCH LABORATORIES

OFFICE DF AEROSPACE RESEARCH

UNITED STATES AIR FORCE

BEDFORD, MASSACHUSETTS 
NEW YORK UNIVERSITY

COURANT INSTITUTE - LIBRARY

251 Mercer Si. New York, N,Y, 10012

Requests for additional copies by agencies of the Department of Defense, their contractors, or other government agencies should be directed to:

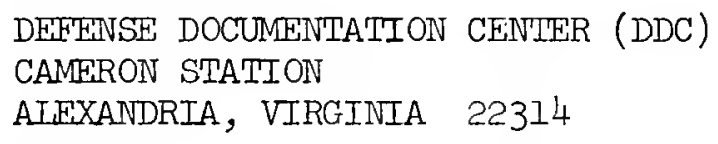

Department of Defense contractors must be established for DDC services or have their "need-to-know" certified by the cognizant military agency of their project or contract.

All other persons and organizations should apply to the:

Clearinghouse for Federal Scientific and Technical Information Sills Building 5285 Port Royal Road Springfield, Virginia 22151 
AFCRI- $65-456$

ASYMPTOTIC EXPANSIONS OF SOLUTIONS OF INITIAL-BOUNDARY

VAIUE PROBIEMS FOR A DISPERSIVE HYFFRBOIIC

EQUATION

Norman Bleistein

Robert M. IEwis

NE'W YORK UNIVERSITY

Courant Institute of Mathematical Sciences

Division of Electromagnetic Research

Contract No. AF 19(628)4065

Project No. 4603

Task No. 460302

Research Report No. EMp-207

June, 1965

Prepared

for:

AIR FORCE CAMBRIDGE RESEARCH IABORATORIES

OFFICE OF AEROSPACE RESEARCH

UNITED STATES AIR FORCE

BEDFORD, MASSACHUSETTS

NEW YORK LINIVERSITY

COURANT INSTITUTE - LIBRARY

251 Mercer St. New York, N.Y. 10012 


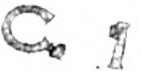




\section{Abstract}

Initial-boundary value problems for an energy conserving dispersive hyperbolic equation, the Klein-Gordon equation, are considered. This equation exhibits the main feature of dispersion: The speed of propagation depends on frequency. Problems in two space dimensions with a parabolic boundary are discussed.

The primary purpose of this paper is to compare the asymptotic expansion of solutions obtained by a technique we call the ray method with the asymptotic expansion of the exact solution. In the cases considered, the solutions agree.

In addition a numerical comparison is made of the exact and asymptotic solutions for a specified region of space time. 

Asymptotic Expansions of Solutions of Initial-Boundary Value Problems for a Dispersive Hyperbolic Equation

M. Bleistein and Robert M. Lewis

\section{(1) Introduction}

In the past few years there has been a great deal of interest in the asymptotic expansion of solutions of boundary value problems and initial-boundary value problems for partial differential equations. In some cases, the "far field" is obtained by asymptotic methods and in other examples the expansion is obtained for large values of a parameter that appears "naturally" in the equation, and/or initial and boundary conditions.

A large class of problems can be treated by methods in which certain curves, called rays, play an essential role. Their importance rests on the fact that on these rays the functions which are used to make up the solution can be shown to satisfy ordinary differential equations which are often solvable.

This "ray method" has been extensively studied by J. B. Keller and his associates at the Courant Institute." A significant advantage of the method is that it eliminates the necessity of first finding the exact solution in order to find the asymptotic expansion. Consequently, many problems for which the exact solution is not known can be treated by the ray method. In particular it has led to a "geometric theory of diffraction" [2].

\footnotetext{
* D. S. Jones [1] refers to this method as "Keller's Method."
} 
Recently, the ray method has been applied to time dependent problems and, in particular, dispersive hyperbolic equations [3]. A particular equation of this type, the Klein-Gordon equation, may be used to describe the propagation of electromagnetic waves in certain plasmas [3]. Initial-boundary value problems for this equation are presently being studied, since it is the simplest example of a large class of dispersive hyperbolic equations.

As of now, there is no general proof that the ray method actualiy yields the asymptotic expansion of the exact solution. The purpose of the research in this paper is to compare the leading term of the asymptotic expansion of the exact solution and the "ray solution" for particular problems. The results are shown to agree and thereby add to the overwhelming evidence that the ray method is valid.

We consider initial-boundary value problems in two space dimensions for the Klein-Gordon equation. The boundary is a parabola and we require that the solution satisfy a homogeneous condition.

The total solution is assumed to consist of a sum of a primary field and a reflected field. The associated rays are called primary and reflected rays and are straight lines in space time. The primary field must satisfy the equation and the initial conditions. The reflected field is taken to be zero initially and is determined at the boundary in such a way that the total solution satisies the given boundary condition. The primary rays are emitted from the plane $t=0$ in space-time. For each primary ray incident on the boundary, a reflected ray is produced. We introduce the notion of group speed and a group velocity vector for the rays. These quantities are "frequency dependent" due to the dispersive nature of the equation being solved. 
Two types of problems are considered. The distinguishing feature is the way in which the initial data depends on the large parameter. In one case, at most two primary rays can emanate from each point in the "initial plane," $t=0$. In the other, an infinite set of primary rays are produced at some points of the initial plane. Their group speeds cover the complete continum of values from zero up to the "characteristic speed" of the equation (see figure 1). The "ray method expansion" of the leading term of the primary and reflected fields are then calculated.

These problems have been so chosen that the solution can also be obtained exactly by time-reducing the equation and solving a partial differential equation in the space variables. This new equation is golvable by using a method introduced by $\mathrm{H}$. Iamb [4] to treat the reduced wave equation in a domain with a parabolic boundary. The solution, $u$, is expressed as a Fourier transform of the solution of the time-reduced problem. This transform can then be expanded asymptotically by standard methods of asymptotic expansion of integrals. In both cases, the solutions obtained by the two methods are seen to agree exactly.

For a particular example, we make a numerical comparison of the far fields of the exact and asymptotic solutions. The results are almost identical for large values of the parameter and agree surprisingly well for smaller values. 
(2) Solution by the Ray Method

(2.1) Sumary of the Ray Nethod

Asymptotic solutions for $\lambda \rightarrow \infty$ of the hyperbolic partial differential equation *

$$
c^{2} u_{x_{v} x_{v}}-u_{t t}-\lambda^{2} b^{2} u=0
$$

are discussed in [3]. The results obtained there are similar to those discussed in [6], but are considerably simpler because $b$ and $c$ are constants in (1). In [3] asymptotic solutions of (1) of the form,

$$
u(t, \underline{X}) \sim \exp [i \lambda s(t, \underline{X})] z(t, \underline{X}) ; \quad \underline{X}=\left(x_{1}, \cdots, x_{n}\right),
$$

are obtained. In this section we summarize the results derived there.

By inserting (2) in (1) one obtains a transport equation for $z$ and a dispersion equation for $\mathrm{s}$. These partial differential equations may then be solved explicitly. In order to describe the solutions $s$ and $z$, we introduce the quantities

$$
k_{v}=s_{x_{v}} \quad \omega=-s_{t} \quad \underline{K}=\left(k_{I} \cdots, k_{n}\right) ; \quad k^{2}=k_{v} k_{v} ;
$$

which satisfy the dispersion relation.

$$
c^{2} k^{2}-w^{2}+b^{2}=0 \text {, or } \omega=h(k)= \pm h_{0}(k) ; h_{0}=\sqrt{c^{2} k^{2}+b^{2}} \text {. }
$$


We also introduce an $n$-parameter family of straight lines in ( $t, X)$-space. These lines are called rays* ${ }^{*}$. In terms of the $n$ parameters $\Sigma=\left(\sigma_{1}, \cdots, \sigma_{n}\right)$ the rays are given by $\underline{X}=\underline{X}(t ; \underline{\Sigma})$ where

$$
x_{v}(t ; \Sigma)=x_{v_{0}}(\Sigma)+\left[t-t_{0}(\Sigma)\right] g_{v}(\Sigma) \text {. }
$$

Here $g_{v}$ is a component of the group velocity vector $G=\left(g_{1}, \cdots, g_{n}\right)$ defined below. Along each ray $\underline{K}$ and $\omega$ have the constant values

$$
\mathrm{k}_{v}=\mathrm{k}_{v_{0}}(\Sigma), \omega=\omega_{0}(\underline{\Sigma}),
$$

which must satisfy (4). Furthermore

$$
g_{v}=g_{v}(\Sigma)=\frac{\partial \omega}{\partial k_{v}}=\frac{c^{2} k_{v}}{\omega}=\frac{c^{2} k_{v_{0}}(\Sigma)}{\omega_{0}(\Sigma)} .
$$

Along each ray, $s(t, \underline{X})$ is given parametrically by

$$
s=s(t)=s[t, \underline{X}(t ; \underline{\Sigma})]=s_{0}(\underline{\Sigma})+[t-\tau(\underline{\Sigma})] \ell(\underline{\Sigma}),
$$

where

$$
\delta=k_{\nu} g_{\nu}-\omega=\frac{c^{2} k^{2}}{\omega}-\omega=-\frac{b^{2}}{\omega}=-\frac{b^{2}}{\omega_{0}(\underline{\Sigma})} \text {. }
$$

Furthermore, $z(t, \underline{X})$ is given parametrically by

* The lines in space-time and their projections in space are called rays. The meaning will be clear in context. 
(10) $z=z(t)=z[t, \underline{X}(t ; \underline{\Sigma})]=z_{0}(\underline{\Sigma})\left[\frac{j\left(t_{0}\right)}{j(t)}\right]^{I / 2} ; z_{0}(\underline{\Sigma})=z\left[t_{0}, \underline{X}_{0}(\underline{\Sigma})\right]$.

Here $j(t)=j(t ; \Sigma)$ is the jacobian of the mapping defined by the rays (5), that is

$$
j(t ; \Sigma)=\operatorname{det} \frac{\partial \mathrm{x}_{\nu}(t ; \Sigma)}{\partial \sigma_{\mu}}
$$

We see that the function $(2,8,10)$ is uniquely determined once the "initial values" $t_{0}, \underline{x}_{0}, s_{0}, \underline{K}_{0}, \omega_{0}$, and $z_{0}$ are given as functions of $\underline{E}$. In general, the values of $t_{0}, \underline{x}_{0}, s_{0}$ and $z_{0}$ on some "initial manifold" $M$ in ( $t, \underline{X}$ )space are derived from the data (initial data, boundary data, etc.) of the given problem for (1). The values of $\underline{K}_{0}$ and $w_{0}$ then follow from (3) and (4). For example, if $M$ is the initial plane $t=0$, and $z(0, \underline{X})=z_{0}(\underline{X})$ and $s(O, \underline{X})=s_{0}(\underline{X})$ are known, we may set $x_{v_{0}}=\sigma_{v} \cdot$ Then

$$
x_{v_{0}}=\sigma_{v}, t_{0}=0, s_{0}=s_{0}(\underline{\Sigma}), z_{0}=z_{0}(\underline{\Sigma})
$$

By differentiation along $M$ we find that*

$$
k_{v_{0}}=\frac{\partial s_{0}}{\partial x_{v}}(\underline{\Sigma}), \quad \omega_{0}= \pm \sqrt{c^{2} \frac{\partial s_{0}}{\partial x_{v}} \frac{\partial s_{0}}{\partial x_{v}}+b^{2}} \text {. }
$$

The values of $s_{0}(\underline{\Sigma})$ and $z_{0}(\Sigma)$ may be determined from the initial data of the given problem, (see section (2.2).). If $M$ is a boundary, given 
parametrically by

(14) $x_{v}=x_{v_{0}}\left(\sigma_{1}, \cdots, \sigma_{n-1}\right), t=T\left(\tau=\sigma_{n}\right)$

the values

(15)

$$
s\left(\tau, \underline{X}_{0}\right)=s_{0}(\Sigma) ; \quad \Sigma\left(\tau, \underline{X}_{0}\right)=z_{0}(\Sigma)
$$

are obtained from the boundary data of the given problem. (See section (2.4).) Then by differentiation along $M$ we find that

(16) $\omega_{0}=-\frac{\partial s_{O}}{\partial \tau}, \quad k_{v_{0}} \frac{\partial x_{v}}{\partial \sigma_{j}}=\frac{\partial s_{0}}{\partial \sigma_{j}} ; j=1, \cdots, n-1$.

Then (16) and (4) provide $n+1$ equations for the $n+1$ quantities

$\omega_{0}, k_{10}, \cdots, k_{n O} \cdot$

In some cases the initial manifold $M$ is of dimension less than $n$. In section (2.3), $n=2$ and $M$ is the (one dimensional) line 
(17)

$$
x_{1}=x_{0}(=\text { constant }), t=0, x_{2}=\sigma_{2} ;
$$

while, on $\mathrm{M}$,

$$
s\left(0, x_{0}, \sigma_{2}\right)=s_{0}(=\text { constant }) \text {. }
$$

Differentiation along $M$ yields

$$
\mathrm{k}_{2}=\mathrm{k}_{20} \equiv 0
$$

Then (19) and (4) provide only two equations for the three quantities $k_{10}, k_{20}, w_{0}$. We may therefore introduce an additional independent parameter, say $k_{10}=\sigma_{1}$, and then $\underline{K}_{0}$ and $u_{0}$ are given by

$$
k_{10}=\sigma_{1}, k_{20}=0, \omega_{0}= \pm \sqrt{c^{2} \sigma_{1}^{2}+b^{2}} .
$$

If, as in the above example, the initial manifold is of dimension less than $n$, it is easy to see that $M$ is a "caustic" of the ray family, i.e. the jacobian $j(t)$ vanishes on $M$. In this case the rolution (10) for $z(t)$ must be modified because $z\left(t_{0}\right)$ is infinite. In [3] it is shown that 


$$
z=z(t)=z[t, \underline{X}(t ; \underline{\Sigma})]=\tilde{z}(\underline{\Sigma}) c[j(t)]^{-1 / 2}
$$

The (finite) value of $\tilde{z}$ nust be obtained by an "indirect method" from the data of the original problem for (1). (See section (2.3).) (The derivation of (21) in [3] is given for the case when $M$ is zero-dimensional, but the argument is valid for any dimension less than $\mathrm{n}$. )

Zero, one, or more rays of the n-parameter family may pass through a given point $(t, \underline{X})$. It is understood that at each point (2) is to be summed over the rays passing through that point. Then (2) is an "asymptotic solution" of (1). The leading term of the asymptotic expansion of the solution of an initial-boundary value problem for (I) then consists of a sum of one or more such asymptotic solutions, so chosen as to satisfy the conditions of the problem. The "ray method" summarized here will be illustrated in sections (2.2), (2.3), (2.5) and (2.6) by applying it to two initial-boundary value problems for (1). In those problems we require the solution of (I) which satisfies the initial conditions

$$
u(0, \underline{X})=u_{0}\left(x_{1} ; \lambda\right) ; u_{t}(0, \underline{X})=u_{1}\left(x_{1} ; \lambda\right)
$$

and the boundary condition

(23) $u=0$ on $B$.

The functions, $u_{0}$ and $u_{1}$, will be specified. The boundary $B$ will be the parabola 
(24) $\quad x_{2}^{2}=-4 p\left(x_{1}-p\right)$.

In terms of the polar coordinates, $\rho=\sqrt{x_{1}^{2}+x_{2}^{2}}, \theta=\arctan x_{2} / x_{1}$, (24) becomes

(25) $\rho(\theta)=\frac{2 p}{1+\cos \theta}$.

We seek the solution $u$ in the exterior of the parabola, defined by $\rho \geq 2 p(1+\cos \theta)^{-1}$. The initial data is assumed to vanish at the boundary. The solution of each problem consists of a sum of solutions,

(26) $u=u_{P}+u_{R} \cdot$

The "primary term" $u_{\mathrm{P}}$ is chosen so as to satisfy the initial conditions of the problem (i.e., the initial conditions for $u$ determine the values, $s_{0}, z_{0}$, etc. for $u_{P}$ on $M_{P}$ which is now the initial plane, $\left.t=0\right)$. The "reflected term" " $R$ is then constructed in such a way that $u$ satisfies the boundary condition. Hence $u_{R}=-u_{P}$ on $B$. (This determines the initial values, $s_{0}$, $z_{O}$, etc. for $u_{R}$ on $M_{R}$ which is now the boundary.) of course the initial conditions must not be "spoiled" by $u_{R}$. This means that the reflected rays, which emanate from the boundary, must not intersect the initial plane. We shall see that these conditions are just enough to determine $u_{R}$ uniquely. 


\section{(2.2) The Primary Field for Oscillatory Initial Data}

Let us consider initial data that is already of the form of (2), that is

$u(0, \underline{X}) \sim z_{0}\left(x_{I}\right) \exp \left[i \lambda_{s_{0}}\left(x_{I}\right)\right], u_{t}(0, \underline{X}) \sim z_{I}\left(x_{I}\right) \exp \left[i \lambda_{s_{I}}\left(x_{I}\right)\right]$.

This will be called oscillatory initial data [5]. It is shown in [3] that this problem can be solved if we can find the solution to the simpler problem where

(27) $u(0, \underline{X}) \sim z_{0}\left(x_{I}\right) \exp \left[i \lambda_{s_{0}}\left(x_{I}\right)\right], u_{t}(0, \underline{X}) \sim-i \lambda h_{0}\left[\left|s_{0}^{\prime}\left(\sigma_{I}\right)\right|\right] z_{0}\left(x_{I}\right) \exp \left[i \lambda s_{0}\left(x_{I}\right)\right]$, with $s_{0}$ and $z_{0}$ given.* Then by comparing (27) and (2) and using (12) and (13) we find that on the initial plane ${ }_{P}$,

(28) $x_{I}=\sigma_{I}, x_{2}=\sigma_{2}, t=0 ; s_{0}=s_{0}\left(\sigma_{I}\right), z_{0}=z_{0}\left(\sigma_{I}\right), k_{10}=s_{0}\left(\sigma_{I}\right), k_{20}=0$. Also, from (20) and (27)

$$
\omega_{0}=\sqrt{c^{2}\left[s_{0} \cdot\left(\sigma_{1}\right)\right]^{2}+b^{2}}=h_{0}\left[\left|s_{0}^{1}\left(\sigma_{1}\right)\right|\right] .
$$

* We use the symbols $s$ and $z$ here for given functions and in section (2.I) for the initial values on rays. Since the given functions will immediately be defined as the initial values on primary rays, this double meaning should cause no difficulty. 
From (5) and (7) it follows that the primary rays are described by the equations

(30) $x_{1}=\sigma_{1}+g_{1}\left(\sigma_{1}\right) t, x_{2}=\sigma_{2} ; g_{1}=c^{2} s_{0}^{1}\left(\sigma_{l}\right) h_{0}^{-I}\left[\left|s_{0}^{\prime}(\sigma)\right|\right]$.

By using (8) and (9) we find that the phase is given by

(3I) $s=s_{0}\left(\sigma_{1}\right)+\ell\left(\sigma_{I}\right) t ; \quad \ell=-b^{2} h_{0}^{-1}\left[\left|s_{0}^{1}\left(\sigma_{1}\right)\right|\right]$.

$z(t)$ is defined by (10). We take $t_{0}=0$ and calculate $j$, defined by (11), from (29). The result is

(32) $z(t)=z_{0}\left(\sigma_{1}\right)\left[1+c^{2} b^{2} s_{0}^{11} h_{0}^{-3} t\right]^{-1 / 2}$

Let us assume that $s_{0}^{\prime \prime}>0$. Then for $t \geq 0$ the jacoibian does not vanish and therefore there are no caustics for $u_{\mathrm{P}^{*}}$. We now use (31) and (32) in $(2)$ and express $u_{P}(t, \underline{X})$ parametrically with parameters $\left(\sigma_{1}, \sigma_{2}\right)$ by (29) and

(33) $u_{P} \backsim z_{0}\left(\sigma_{1}\right)\left[1+c^{2} b^{2} s_{0}^{\prime \prime} h_{0}^{-3} t\right]^{-1 / 2} \exp \left[i \lambda\left\{s_{0}\left(\sigma_{1}\right)-b^{2} h_{0}^{-1} t\right\}\right]$.

As noted in the introduction, to find $u_{P}(t, X)$ we add up all contributions of the form (32) from rays passing through $(t, X)$.

\section{(2.3) The Primary Field for Rapidly Varying Initial Data}

We now consider initial data of the form 
(34) $u(0, \underline{X})=u_{0}\left[\lambda\left(x_{1}-x_{0}\right)\right], u_{t}(0, X)=\lambda u_{1}\left[\lambda\left(x_{1}-x_{0}\right)\right] ; x_{0}>p$

and assume that $u_{0}(\xi)$ and $u_{1}(\xi)$ have compact support. ${ }^{*}$ Then as $\lambda \rightarrow \infty$ the support ${ }^{*}$ of the injilal data shrinks to the line $L\left(t=0, x_{1}=x_{0}\right)$. In this case we assume that the rays emanate from $L^{* *}$ (i.e. $L=M_{P}$ ) and that $s$ is constanton $L$ (i.e. independent of $\left.x_{2}=\sigma_{2}\right)$. Then (19) holds. The choice of sign in the dispersion relation (4) is not dictated by the initial data. Since equation (1) is linear, we may take

(35) $u_{p}=u_{+}+u_{-} ; u_{ \pm} \sim z^{ \pm} \exp \left[i \lambda s^{+}\right]$,

where the choice of sign is to be identified with the choice in (4). We apply the method outlined in the introduction for the case of a lower dimensional manifold $M$ and find that the rays, $s^{ \pm}$and $z^{ \pm}$are given by

\footnotetext{
* The support of a function is the range of values for which the function is not zero. A function is said to have compact support if its support is bounded.

* This assumption is intuitively reasonable. It is fully justified when the solution obtained is compared to the asymptotic expansion of the exact solution and they are found to agree.
} 
(36) $x_{1}=x_{0}+c^{2} k_{1} h_{0}^{-1} t, x_{2}=\sigma_{2}$;

(37) $s^{ \pm}=s_{0}^{ \pm} \mp b^{2} h_{0}^{-1} t$;

(38) $z^{ \pm}=\zeta^{ \pm} t^{-1 / 2}$.

H:re we have used equations (5), (8), (11) and (21) with $\zeta^{ \pm} t^{-I / 2}=z^{ \pm}(\underline{\Sigma})[j(t)]^{-I / 2}$. The constant $s_{0}^{ \pm}$and the function $\zeta^{ \pm}$are determined by the indirect method discussed in [3]: We first obtain the exact solution of the initial value problem (1), (33) (with no boundary). The asymptotic expansion of this solution is then compared with our result $u_{p}$ given by $(35)-(37)$ and $s_{0}^{ \pm}$and $\zeta^{ \pm}$can be identified. The results obtained by this method are

(39) $\mathrm{s}_{0}^{ \pm}=0,5^{ \pm}=\left(\frac{2 \pi}{\lambda}\right)^{1 / 2} a_{ \pm}\left(\sigma_{1}\right) e^{\bar{\tau} \frac{\dot{i} \pi}{4}}\left(h_{0}^{\prime \prime}\left(\sigma_{1}\right)\right)^{-1 / 2}$;

(40) $a_{ \pm}\left(\sigma_{1}\right)=\frac{1}{4 \pi} \int_{-\infty}^{\infty}\left[u_{0}(\xi) \pm \frac{i}{h_{0}\left(\sigma_{1}\right)} u_{1}(\xi)\right] \exp \left[-i \sigma_{1} \xi\right] d \xi$,

(4I) $h_{0}^{\prime \prime}\left(\sigma_{1}\right)=c^{2} b^{2} h_{0}^{-3}$.

Then $u_{P}(t, X)$ is expressed parametrically with parameters $x_{2}=\sigma_{2}, k_{I}=\sigma_{I}$ by (35) and

(42) $u_{P} \backsim \sum_{ \pm} \zeta^{ \pm} t^{-1 / 2} \exp \left[\mp i \lambda b^{2} h_{0}^{-1} t\right]$. 
(2.4) The Reflected Field at the Boundary

In this section we shall show how the values of $s_{0}, z_{0}, \omega_{0}, \underline{K}_{0}$ and $G_{0}$ for $u_{R}$ are determined on the boundary $M_{R}$. For parameters, we choose $\Sigma=(\tau, \theta)$ and using $(24)$, describe $M_{R}$ parametrically by the equations

(43) $\mathrm{t}=\tau, \rho(\theta)=\frac{2 p}{1+\cos \theta} ; \underline{X}(\theta)=\rho(\theta)(\cos \theta, \sin \theta)$.

$$
0 \leq \tau,-\pi \leq \theta \leq \pi
$$

With $u_{P}$ and $u_{R}$ of the form in (2) we find, by applying the boundary condition (22), that*

(44) $z_{P}[\tau, \underline{X}(\theta)]=-z_{R}[\tau, \underline{X}(\theta)]$

(45) $s_{P}[\tau, \underline{X}(\theta)]=s_{R}[\tau, \underline{X}(\theta)]$.

Differentiation of (45) with respect to $\tau$ and $\theta$ reveals that

(46) $\omega_{P}(\tau, \theta)=\omega_{R}(\tau, \theta)$,

(47) $k_{I P}(\tau, \theta) \dot{x}_{I}+k_{2 P}(\tau, \theta) \dot{x}_{2}=k_{I R}(\tau, \theta) \dot{x}_{I}+k_{2 R}(\tau, \theta) x_{2}$,

where $\left({ }^{\bullet}\right)$ denotes differentiation with respect to $\theta$. Then from (46)

* The subscripts $P$ and $R$ are used throughout this discussion to differentiate between quantities associated with the primary field $u_{p}$ and the reflected field $u_{R}$. 
and (4), we conclude that

(48) $k_{P}^{2}=k_{R}^{2}$.

We define $\alpha_{P}(\tau, \theta)$ as the angle of incidence of a primary ray.

It is the angle between $\underline{K}_{P}$ and the normal to $B$ at the point $(\tau, \underline{X}(\theta))$. We define $\alpha_{R}$ in an analogous manner as the angle of reflection. (47) and (48) show that $\underline{K}_{P}$ and $\underline{K}_{R}$ have the same length and projection on the tangent vector to $B, \underline{\dot{X}}(\theta)$. It follows that $\cos \alpha_{P}=\cos \alpha_{R^{0}}$ If also $\sin \alpha_{P}=\sin \alpha_{R}$, the reflected rays would coincide with the primary rays. (See figure (1).) In this case they would intersect the initial plane and $u_{R}$ would be non-zero for $t=0$. Then $u=u_{P}+u_{R}$ would no longer satisfy the initial conditions. Thus we conclude that $\sin \alpha_{P}=-\sin \alpha_{R}$

Equations (19) and (28) show that (for both types of initial data), $k_{2 P}=0$, $i \cdot e$. the primary rays are parallel to the axis of the parabola. Then it is easy to show that

(49) $\quad \mathbb{K}_{R}(\tau, \theta)=-k_{I P}(\cos \theta, \sin \theta)$,

that is, the reflected rays would intersect the focus of the parabola if extended backwards. This is a well know property of the parabola. Finally, (7), (46) and (49) yield

(50) $G_{R}(\tau, \theta)=-c^{2} k_{1 P}(\tau, \theta) \omega_{P}^{-1}(\tau, \theta)(\cos \theta, \sin \theta)$. 


\section{(2.5) The Reflected Field for Oscillatory Initial Data}

We consider again the problem introduced in section (2.1) with the initial data of section (2.2). For values of $\sigma_{1}$ for which $s_{0}^{\prime}\left(\sigma_{1}\right)<0$, the primary rays given by (30) will be incident at the boundary. By using (30) and (43) we find that

$$
\begin{aligned}
& x_{I}(\tau, \theta)=\frac{2 p}{1+\cos \theta}=\sigma_{1}+g_{1} \tau ; x_{2}(\tau, \theta)=\frac{2 p \sin \theta}{1+\cos \theta}=\sigma_{2} ; \\
& g_{I}=c^{2} s_{0}^{1}\left(\sigma_{I}\right) h_{0}^{-2}\left[\left|s_{0}^{1}\left(\sigma_{1}\right)\right|\right]
\end{aligned}
$$

We recall that our assumption $s_{0}^{\prime \prime}>0$ led to the conclusion that the jacobian defined by (II) and (30) does not vanish. It follows that (50) always has a unique solution for $\left(\sigma_{1}, \sigma_{2}\right)$ in terms of $(\tau, \theta)$. Thus each reflected ray is associated with a unique primary ray. By using (5), (50) and (51) we find that

(52) $x=\rho(\cos \theta, \sin \theta) ; \quad \rho=\frac{2 p}{I+\cos \theta}-\frac{c^{2} s_{0}^{\prime}\left(\sigma_{I}\right)}{h_{0}\left[\left|s_{0}^{\prime}\left(\sigma_{I}\right)\right|\right]} \cdot(t-\tau)$. Using (8), (31) and (45) it follows that

(53) $s_{R}=s_{P}(\tau, \theta)-b^{2} h_{0}^{-I}(t-\tau)=s_{0}\left(\sigma_{I}\right)-b^{2} h_{0}^{-1} t$. 
The jacobian $j_{R}$, defined by (II) with $\Sigma=(\tau, \theta)$, can be calculated from (52). The quotient needed for (10) is given by

(54) $\frac{j_{R}(\tau)}{j_{R}(t)}=\frac{2 p}{\rho(1+\cos \theta)}\left[\frac{1+c^{2} b^{2} s_{0}^{n} h_{0}^{-3}}{1+c^{2} b^{2} s_{0}^{n} h_{0}^{-3}}\right]$.

We use (10), (32) and $(44)$ to determine $\Sigma_{R}$. This yields

(55) $z_{R}=-z_{0}\left(\sigma_{I}\right)\left[\frac{2 p}{\rho(1+\cos \theta)}\right]^{I / 2}\left[I+c^{2} b^{2} s_{0}^{11 h} h_{0}^{-3} t\right]^{-1 / 2}$.

When our results are substituted in (2) $u_{R}$ is given parametrically with parameters $(\tau, \theta)$ by $(52)$ and

(56) $u_{R} \sim-z_{0}\left(\sigma_{I}\right)\left[\frac{2 p}{\rho(1+\cos \theta)}\right]^{I / 2}\left[1+c^{2} b^{2} s_{0}^{\prime \prime} h_{0}^{-3} t\right]-1 / 2 \exp \left[i \lambda\left\{s_{0}\left(\sigma_{I}\right)-b^{2} h_{0}^{-1} t\right\}\right]$.

Here $\sigma_{1}$ must be determined from (5I) as a function of $(\tau, \theta)$.

\section{(2.6) The Reflected Field for Rapialy Varying Initial Data.}

We consider again, the problem of section (2.1) with the initial data of section (2.3). The only primary rays incident at the boundary are those for which $g_{1}$, given by (30), is negative. By using (36) and (43) we find that the analogue of equation (50) for this evanple is 
(57) $x_{1}(\tau, \theta)=\frac{2 p \cos \theta}{1+\cos \theta}=x_{0} \pm \frac{c^{2} k_{1}}{h_{0}} \tau, \pm k_{1}<0 ; x_{2}=\frac{2 p \sin \theta}{1+\cos \theta}=\sigma_{2}$. This equation always has a solution forthe parameters $\left(k_{1}, \sigma_{2}\right)$ of the primary field in terms of $(\tau, \theta)$, the parameters of the reflected field. The calculation of $u_{R}$ can now be carried out exactly as in section (2.5) and therefore we omit the details. The rays are given by

(58) $\underline{X}=\rho(\cos \theta, \sin \theta) ; \rho=\frac{2 p}{1+\cos \theta}+\frac{c^{2}\left|k_{1}\right|}{h_{0}}(t-\tau)$. $u_{R}(t, \underline{X})$ is given parametrically by $(58)$ and

(59) $u_{\mathrm{R}} \sim \sum_{ \pm}-\zeta^{ \pm}\left[\frac{2 p}{\rho(1+\cos \theta) t}\right]^{I / 2} \exp \left[\overline{+} i \lambda b^{2} h_{0}^{-1}\left(k_{I}\right) t\right]$.

Here $\zeta^{ \pm}$are given by (38)-(40), $h_{0}$ is given by (6) and $h_{l}$ can be calculated. as a function of $(\tau, \theta)$ from (57). 


\section{(3) Asymptotic Expansion of the Exact Solution}

(3.1) Derivation of the Solution

In this section we again consider the initial boundary value problems discussed in section (2). Our goal is to obtain the leading term of the asymptotic expansion of the exact solution in order to compare it with the results obtained by the ray method. As in section (2) we assume that $u=u_{P}+u_{R}$. The primary field $u_{P}$ is the solution of an initial-value problem (without a boundary). The reflected field $u_{R}$ is the solution of an initial-boundary value problem, with zero initial data and boundary data chosen in such a way that $u=0$ on $B$.

To find $u_{P}$ and $u_{R}$, we first separate variables by applying Fourier transform with respect to $t$. This reduces the problem to solving partial differential equations in $\underline{X}$ for functions which will be called $v_{P}$ and $v_{R}$. When these new functions are known, their inverse transforms yield $u_{P}$ and $u_{R}$, expressed as integrals. By expanding these integrals asymptotically, we obtain the asymptotic expansion of $u(t, \underline{X})$.

For both $u_{P}$ and $u_{R}$ we define

$$
v(\underline{X}, \omega)=\int_{0}^{\infty} u(t, \underline{X}) \exp [i \lambda \omega t] d t ; \operatorname{Im} \omega \geq 0
$$

and then

$$
u(t, \underline{X})=\sum_{ \pm} \frac{\lambda}{2 \pi} \int_{0}^{\infty} v(\underline{X}, \pm \omega) \exp [\mp i \lambda \omega t] d \omega .
$$

$u_{P}$ is a solution of the initial value problem (2.1), (2.21) and is independent of $x_{2}$ since (2.21) is independent of $x_{2}$. In this case $v_{P}$ is a function only of $x_{1}$. By taking the Fourier transform of (2.I) and 
using (2.21), it follows that $v_{p}$ must satisfy the equation

$$
v_{p}^{\prime \prime}\left(x_{I}, \omega\right)+\lambda^{2} c^{-2}\left(\omega^{2}-b^{2}\right) v_{p}\left(x_{I}, \omega\right)=c^{-2} r\left(x_{I} \omega ; \lambda\right) ;
$$

(4)

$$
r\left(x_{1}, \omega ; \lambda\right)=i \lambda \omega u_{0}\left(x_{1} ; \lambda\right)-u_{1}\left(x_{1} ; \lambda\right)
$$

$v_{p}$ also satisfies the "radiation condition,"

$$
\lim _{\left|x_{1}\right| \rightarrow \infty} v_{P}\left(x_{1}, \omega\right)=0 ; \operatorname{Im} \omega>0 \text {, }
$$

which can be derived as in [6] under the assumption that $u_{0}$ and $u_{1}$ have compact support in $x_{I}$. Analogous to (3), $v_{R}$ must satisfy the equation

$$
\Delta v_{R}(x, \omega)+\lambda^{2} c^{-2}\left(\omega^{2}-b^{2}\right) v_{R}(x, \omega)=0 ; \Delta=\frac{\partial^{2}}{\partial x_{1}^{2}}+\cdot \frac{\partial^{2}}{\partial x_{2}^{2}} .
$$

The boundary $B$ is given by (2.23) or (2.24) and the boundary condition (22) leads to

$$
v_{P}+v_{R}=0 \text { on } B \text {. }
$$

When $v_{P}$ is known, (7) defines $v_{R}$ on $B$. Analogous to (5) we have the condition

$$
\lim _{\rho \rightarrow \infty} v_{R}(\underline{x}, \omega)=0 ; \operatorname{Im} u>0: \rho=\left(x_{1}^{2}+x_{2}^{2}\right)^{\frac{1}{2}}
$$

Since (3) is an ordinary differential equation with constant coefficients the solution to (3), (4), (5) can immediately be determined. 
The result for $\omega^{2}>b^{2}$ is

(9)

$$
\begin{aligned}
& v_{P}\left(x_{1}, \pm \omega\right)= \frac{1}{2 i \lambda c \sqrt{\omega^{2}-b^{2}}}\left[\int_{-\infty}^{x_{1}} r(\sigma, \pm \omega ; \lambda) \exp \left[ \pm i \lambda k\left(x_{1}-\sigma\right)\right] d \sigma\right. \\
&\left.+\int_{x_{1}}^{\infty} r(\sigma, \pm \omega ; \lambda) \exp \left[\mp i \lambda k\left(x_{1}-\sigma\right)\right] d \sigma\right] ; \\
& c k=\sqrt{\omega^{2}-b^{2}} .
\end{aligned}
$$

The solution of (9) for $\omega^{2}<b^{2}$ could be obtainea by analytic continuation of (9), but is of no interest since it is exponentially small in $\lambda$ and leads to an exponentially small contribution to $u_{P}$. At the boundary, the first integral in (9) is zero, since the entire domain of integration is outside the support of $r(\sigma, \omega ; \lambda)$. Therefore, for $\omega>b$,

$$
v_{P}\left(x_{1}, \pm \omega\right)=A( \pm \omega) \exp \left[\mp i \lambda k x_{1}\right] \text { on } B \text {, }
$$

where

$$
A( \pm \omega)= \pm \frac{1}{2 i \lambda c \sqrt{\omega^{2}-b^{2}}} \int_{-\infty}^{\infty} r(\sigma, \pm \omega ; \lambda) \exp [ \pm i \lambda i \sigma \sigma] d \sigma .
$$

In order to solve (6) - (8) we use the method of Lamb [6]. We assume that $v_{R}=w \exp \left[\mp i \lambda k x_{l}\right]$. The function $w$ can then be determined in terms of parabolic coordinates $(\xi, \eta)$, which are defined by the equation

$$
(\xi+i \eta)^{2}=\lambda k\left(x_{1}+i x_{2}\right) .
$$

In terms of the polar coorainates, $(\rho, \theta)$, it can be shown that

$$
\xi=\left(\lambda k_{\rho}\right)^{\frac{1}{2}} \cos \frac{\theta}{2}, \eta=(\lambda k \rho)^{\frac{1}{2}} \sin \frac{\theta}{2} .
$$


From (2.24), the boundary is now defined by the equation

(14)

$$
\xi=\xi_{\mathrm{B}}=(\lambda k \mathrm{p})^{\frac{1}{2}}
$$

The calculation of $v_{R}$ is carried out in Appendix I.

The result is

$$
v_{R}(X, \pm \omega)=-A( \pm \omega) \exp \left[\mp i \lambda k x_{1}\right]\left\{E^{ \pm}\left[(\lambda k p)^{\frac{1}{2}}\right]\right\}\left\{E^{ \pm}\left[(\lambda k \rho)^{\frac{1}{2}} \cos \frac{\theta}{2}\right]\right\}
$$

$$
E^{ \pm}(\xi)=\int_{\xi}^{\infty} \exp \left[ \pm 2 i \zeta^{2}\right] d \zeta
$$

This expression can be simplified by expanding $E^{ \pm}$asymptotically for large $\lambda$. By integrating by parts in (16), we obtain

$$
E^{ \pm}(\xi) \sim \frac{\mp \exp \left[ \pm 2 i \xi^{2}\right]}{4 i \xi}
$$

When this result and the half angle formula for $\cos \frac{\theta}{2}$ are substituted in (15), $v_{R}$ can be rewritten as

$$
\mathrm{v}_{\mathrm{R}}(\underline{x}, \pm \omega) \sim-\mathrm{A}( \pm \omega)\left(\frac{2 \rho}{\rho(1+\cos \theta)}\right)^{\frac{1}{2}} \exp [ \pm i \lambda k(\rho-2 p)] \text {. }
$$

\section{(3.2) A Sample Solution}

With $v_{P}$ and $v_{R}$ given by (9) and (18), $u_{P}$ and $u_{R}$ can be determined by substituting in (2) and then expanding asymptotically by the method of stationary phase. This has been carried out for the two problems considered in section (2) and in both cases the results agree exactly with those obtained by the ray method. As an example we consider $u_{R}$ for the problem with oscillatory initial data (2.26). Using that equation and (4) we find that 
(19)

$$
r\left(x_{1}, \pm \omega ; \lambda\right)=i \lambda_{z_{0}}\left(x_{1}\right)\left[ \pm \omega+h_{0}\left(\left|s_{0}^{1}\left(x_{1}\right)\right|\right)\right] \exp \left[i \lambda_{s_{0}}\left(x_{1}\right)\right] .
$$

When this value is substituted into (11),

$A( \pm \omega)= \pm \frac{1}{2 c \sqrt{\omega^{2}-b^{2}}} \int_{-\infty}^{\infty} z_{0}(\sigma)\left[ \pm \omega+h_{0}\left(\left|s_{0}^{i}(\sigma)\right|\right)\right] \exp \left[i \lambda\left\{s_{0}(\sigma) \pm k_{0} \sigma\right] d \sigma\right.$,
$c k=\sqrt{\omega^{2}-b^{2}}$

and then $v_{R}(X, \pm \omega)$ is given by (18) with this value of $A( \pm \omega) . u_{R}(t, \underline{X})$ is now given asymptotically by (2) with $v$ replaced by $v_{R}$ :

$$
\begin{aligned}
u_{R}(t, \underline{X}) \sim \sum_{ \pm} \mp & \frac{\lambda}{4 \pi c^{2}}\left(\frac{2 p}{\rho(1+\cos \theta)}\right)^{\frac{1}{2}} \int_{-\infty}^{\infty} d \sigma \int_{0}^{\infty} d_{u}\left[\frac{z_{0}(0)\left[ \pm \omega+h_{0}\left(\left|s_{0}^{\prime}(0)\right|\right)\right]}{k}\right. \\
& \left.\cdot \exp \left[ \pm i \lambda\{k(\rho-2 p+\sigma)-\omega t\}+i \lambda s_{0}(0)\right]\right] .
\end{aligned}
$$

This integral can be evaluated by the method of stationary phase in two dimensions [3]. We define

$$
\varphi_{ \pm}(\omega, \sigma)= \pm\left\{\frac{\sqrt{\omega^{2}-b^{2}}}{c}(\rho+\sigma-2 p)-\omega t\right\}+s_{0}(\sigma) .
$$

It is necessary to determine the first and secona derivatives of $q_{ \pm}$ with respect to $\omega$ and $\sigma$; these functions are:

(23) $\frac{\partial \varphi_{ \pm}}{\partial \omega}= \pm\left\{\frac{\omega}{c \sqrt{\omega^{2}-b^{2}}}(\rho+\sigma-2 p)-t\right\} ; \quad \frac{\partial \varphi_{ \pm}}{\partial \sigma}= \pm \frac{\sqrt{\omega^{2}-b^{2}}}{c}+s_{0}^{\prime}(\sigma)$.

(24) $\frac{\partial^{2} \varphi_{ \pm}}{\partial \omega^{2}}=\mp\left\{\frac{b^{2}}{c\left(\omega^{2}-b^{2}\right)^{3 / 2}}(\rho+\sigma-2 p)\right\} ; \frac{\partial^{2} \varphi_{ \pm}}{\partial \sigma^{2}}=s_{0}^{\prime \prime}(\sigma)$; 
(25)

$$
\frac{\partial^{2} \varphi_{ \pm}}{\partial \omega d \sigma}= \pm \frac{\omega}{c \sqrt{\omega^{2}-b^{2}}}
$$

The stationary points of $\varphi_{ \pm}$are determined by setting both derivatives in (23) equal to zero. This yields the equations

$$
(\rho+\sigma-2 p)=\frac{c \sqrt{\omega^{2}-b^{2}}}{\omega} t ; \quad c s_{0}^{\prime}(\sigma)=\mp \sqrt{\omega^{2}-b^{2}} .
$$

By solving for $w$ we find that

$$
w=c^{2} s_{0}^{2}(\sigma)+b^{2}=h_{0}\left[\left|s_{0}^{\prime}(\sigma)\right|\right]
$$

Hence, for the lower sign in (21) the integrand is zero. For the upper sign, from (26), it is necessary that $s_{0}(\sigma)<0$. For the domain of the problem $\rho \geq p$. In the support of $z_{0}, \sigma>p$. Therefore in the domain where the integrand is non-zero, $\rho+\sigma-2 p>0$, and the first equation of (26) can always be satisfied for $t>0$. If we define the boundary in space time by $(2.25)$ and $t=\tau$ then at the boundary (26) becomes

$$
\left(\frac{2 p}{1+\cos \theta}+\sigma-2 p\right)=\frac{c \sqrt{\omega^{2}-b^{2}}}{\omega} \tau \text {. }
$$

Multiplying (27) by $\cos \theta$ yields the first equation of (2.j1) which defines $\sigma$ (or $\sigma_{1}$ of section (2)) as a function of $(\tau, \theta)$. The equation for $\sigma_{2}=x_{2}=\rho(\theta) \sin \theta$ in (2.51) is an immediate consequence of (2.25). We see from (26), (27) and (28) that the stationary phase conditions are equivalent to the equation of the rays (2.52), the dispersion relation (2.29) and the relation between the parameters $\left(\sigma_{1}, \sigma_{2}\right)$ and $(\tau, \theta)$. 
We define

(29)

$$
\left(\partial^{2} \varphi\right)=\left(\begin{array}{cc}
\varphi_{\omega \omega} & \varphi_{\omega \sigma} \\
\varphi_{\sigma \omega} & \varphi_{\sigma \sigma}
\end{array}\right)
$$

where all derivatives are evaluated at the stationary point defined by (28). Then

$$
\operatorname{det}\left(\partial^{2} \varphi_{+}\right)=-\frac{h_{0}^{2}}{c^{4} s_{0}^{2}}\left[1+\frac{c^{2} b^{2} s_{0}^{\prime \prime}}{n_{0}^{3}} t\right] .
$$

For $s_{0}>0$, the eigenvalues of $\partial^{2} \varphi$ must be of opposite sign for all $t>0$ and therefore $\operatorname{sig}\left(\partial^{2} \varphi\right)=0$. Evaluating $\varphi$ at the stationary point, we find that

$$
\phi(\omega, \sigma)=-\frac{\mathrm{b}^{2}}{\mathrm{~h}_{0}} t+\mathrm{s}_{0}(\sigma)
$$

The solution is now obtained by using our results in the formula in

[3] for evaluation of integrals by the method of stationary phase in two dimensions. $u_{R}(t, X)$ is given parametrically by $(26),(27)$, and

$$
u_{R} \sim-\left(\frac{2 p}{\rho(1+\cos \theta)}\right)^{\frac{1}{2}} \cdot \frac{z_{0}(\sigma)}{\left[1+c^{2} b^{2} s_{0}^{1 " n_{0}-3} t\right]^{\frac{1}{2}}} \cdot \exp \left[i \lambda\left\{s_{0}(\sigma)-b^{2} h_{0}^{-1} t\right\}\right] .
$$

This is the same result as was obtained in section (2.5) by the rav method. 


\section{(4) Numerical Comparison of the Exact and Asymptotic Solutions}

We consider the problem for $u$ defined in section (2.1) in the special case where

$$
u(0, \underline{X})=0, u_{t}(0, \underline{X})=\delta\left(x_{I}-2 p\right)
$$

In this section we shall compare numerically the exact solution and asymptotic solutions $u_{R}$ for some region of space-time. It is most convenient and probably most interesting to make this comparison on the axis of the parabola for $x_{1} \gg 1$ and $t \gg 1$; i.e., we take $x_{2}=0, x_{1}=\rho=\operatorname{nct}(0<n<I)$, $\theta=0$ and compare the exact and asymptotic solutions as $t \rightarrow \infty$. Let us define $\tilde{u}_{R}$ to be the asymptotic solution obtained by the ray method. $\left(u_{R} \sim \widetilde{u}_{R}\right.$ for $\lambda \rightarrow \infty$.) We also define $\hat{u}_{R}$ to be the expansion of the exact solution for large values of $t . \quad\left(u_{R} \sim \hat{u}_{R}\right.$ as $t \rightarrow \infty, x_{2}=0, x_{1}=$ nct. $)$ In addition we introduce $\hat{\tilde{u}}_{R}$ as the expansion of $\tilde{u}_{R}$ as $t \rightarrow \infty$. $\hat{\tilde{u}}_{R}$ and $\hat{u}_{R}$ will be compared for a typical value of $n$ as $\lambda$ varies.

We note that $\delta\left(x_{1}-2 p\right)=\lambda \delta\left(\lambda\left[x_{1}-2 p\right]\right)$ so that $(1)$ is a special case of rapidly varying initial data. $\tilde{u}_{k}$ is given parametrically by (2.58) and (2.59). $5^{ \pm}$are obtained by using (1), (2.34), (2.39) and (2.40). It then follows that

(2) $\tilde{u}_{R}(t, \rho, 0) \sim \frac{\sum}{ \pm}-\left(\frac{p h_{0}}{8 \pi \lambda c^{2} b^{2} \rho t}\right)^{I / 2} \exp \left[\mp i \lambda b^{2} h_{0}^{-1} t \pm \frac{i \pi}{4}\right]$;

$$
\rho=p+\frac{c^{2}\left|k_{1}\right|}{h_{0}}(t-\tau)
$$


The dependence on $P$ in (2) can be eliminated by making use of (3). Before doing so, we note from (2.57) with $x_{0}=2 p$ that

(4)

$$
\mathrm{p}=\frac{c^{2}\left|k_{I}\right|}{h_{0}} \tau
$$

so that (3) becomes

(5) $\quad p=$ nct; $n=\frac{c\left|k_{1}\right|}{h_{0}}<1$.

Then (2) may be rewritten as

(6) $\tilde{u}_{R}(t, v t, 0) \sim \sum_{ \pm}-\left(\frac{p h_{0}}{8 \pi \lambda b^{2} c^{3} n t^{2}}\right)^{\frac{1}{2}} \exp \left[\mp i \lambda b^{2} h_{0}^{-1} \pm \frac{i \pi}{4}\right]$.

To find $\hat{\vec{u}}_{R}$ we must expand the coefficient of the exponent in (6) in powers of $l / t$. But we see at once that this coefficient is already of the form const. $\frac{1}{t}$. Therefore $\hat{\tilde{u}}_{R}=\tilde{u}_{R}$.

The exact expression for $u_{R}$ can be obtained by using (3.15) with $\theta=0$ and $x_{1}=p . A( \pm \omega)$ is defined by (3.11), where

(7) $r(\sigma, \pm \omega ; \lambda)=-\delta\left(x_{1}-2 p\right)$

as determined by $(I)$ and $(3.4)$. We seek the value of $v_{R}$ for large $p$ so that $\mathrm{E}^{ \pm}\left(\left[\lambda_{\mathrm{k}} \rho\right]^{\frac{1}{2}}\right)$, appearing in $(3.15)$ can be expanded asymptotically for large argument. This expansion can be obtained from (3.17) and the resulting solution for $v_{R}$ is

(8) $V_{R}(\rho, 0, \pm \omega) \sim\left(8 \lambda c^{2} k \sqrt{\lambda k \rho} E^{ \pm}(\sqrt{\lambda k p})\right)^{-1} \exp [ \pm i \lambda(k(\rho+2 p))]$; $c k=\sqrt{\omega^{2}-b^{2}}$. 
Using (3.2) with $u$ replaced by $u_{R}$ and $\rho=$ nct, we find that

(9) $u_{R}(t, v t, 0) \sim \sum_{ \pm} \frac{1}{16 \pi c^{2}} \int_{0}^{\infty} \frac{\exp [ \pm i \lambda(k n c-\omega) t \pm 2 i \lambda k p]}{k \sqrt{\lambda k n c t} E^{ \pm}(\sqrt{\lambda k p})} d \omega, t \rightarrow \infty$.

This integral can be expanded asymptotically by the method of stationary phase for large values of $t$. The result, $\hat{u}_{R}$, is given by

$$
\text { (10) } \begin{gathered}
\hat{u}_{\mathrm{R}} \sim \sum_{ \pm}-\mathrm{R}_{ \pm}\left(\frac{\mathrm{ph}}{8 \pi \lambda \mathrm{b}^{2} \mathrm{c}^{3} \mathrm{nt} \mathrm{t}^{2}}\right)^{\frac{1}{2}} \exp \left[\mp i \lambda b^{2} h_{0}^{-1} t+\frac{i \pi}{4}\right] ; \\
\mathrm{n}=\frac{c k}{h_{0}} \leq 1,
\end{gathered}
$$

where

$$
\text { (11) } \mathrm{R}_{ \pm}=\mp \frac{\exp [ \pm 2 i \lambda k p]}{4 i \sqrt{\lambda k p} E^{ \pm}(\sqrt{\lambda k p})} \text {. }
$$

Comparing (6) and (II) we see that $\widetilde{u}_{R}$ and $\hat{u}_{R}$ differ only in the factors $\mathrm{R}_{ \pm}$. To compare the solutions numerically then, we need only examine $\mathrm{R}_{ \pm}$ and see how they compare to one. It should be noted that $\mathrm{R}_{ \pm}$are complex conjugates and are just ratios of the asymptotic expansions of $\mathrm{E}^{ \pm}(\sqrt{\lambda \mathrm{kp}})$ to the functions themselves. In terms of $n$, we find that

$$
\lambda k p=\frac{\lambda b p}{c} \frac{n}{\sqrt{1-n^{2}}},
$$

so that $R_{ \pm}$are functions of the two (dimensionless) parameters $n$ and $\frac{\lambda b p}{c}$. 
As an example, in figure ( 3 ) we show a graph of the magnitudes of $R R_{+}$and for $I m R_{+}$for $n=\frac{1}{2}$ and $\frac{\lambda b p}{c}=v$ varying. The table below lists some sample values used to construct the graph.

$$
\begin{array}{|c|c|c|}
v & \operatorname{Re} R_{+} & I_{+} R_{+} \\
\pi & 1.0269 & .12338 \\
2 \pi & 1.0083 & .06660 \\
4 \pi & 1.0014 & .03475 \\
8 \pi & 1.0006 & .01686 \\
10 \pi & 1.0004 & .01414
\end{array}
$$




\section{Appendix I}

In this section we will show how the function $v_{R}(\underline{x}, \pm \omega)$, defined in section ( 3.1 ), is obtained by Lamb's method. This function must satisfy the equation

(1)

$$
\Delta v_{R}+\lambda^{2} c^{-2}\left(\omega^{2}-b^{2}\right) v_{R}=0
$$

We assume that

(2) $\quad v_{R}(\underline{X}, \pm \omega)=w(\underline{X}, \pm \omega) \exp \left[\bar{f} i \lambda k x_{1}\right] ; \quad c k=\left(\omega^{2}-b^{2}\right)^{1 / 2}$

Then at the boundary from $(3.10)$

(3)

$$
w(\underline{X}, \underline{t} \omega)=-A( \pm \omega)
$$

In order that $v_{R}$ satisfy (I), w must satisfy the equation,

$$
\Delta w(\underline{X}, \underline{t w}) \mp 2 i \lambda k w_{x_{I}}=0
$$

Following Lamb's example, we introduce the parabolic coordinates, $(\xi, \eta)$, defined by the equation

$$
(\xi+i \eta)^{2}=\lambda k\left(x_{1}+i x_{2}\right)
$$

In terms of the polar coordinates $(\rho, \theta),(5)$ can be reduced to

$$
\xi=(\lambda \mathrm{k} \rho)^{1 / 2} \cos \frac{\theta}{2}, \quad \eta=(\lambda k \rho)^{1 / 2} \sin \frac{\theta}{2} .
$$


From (2.24), the boundary is now defined by the equation

(7)

$$
\xi=\xi_{\mathrm{B}}=(\lambda \mathrm{kp})^{1 / 2} \text {. }
$$

For the function

$$
W(\xi, \eta, \pm w)=w(X(\xi, \eta), \pm w),
$$

the following equation is obtained from (4):

$$
\frac{\partial^{2} W}{\partial \xi^{2}}+\frac{\partial^{2} W}{\partial \eta^{2}} \mp 4 i\left(\xi \frac{\partial}{\partial \xi}-\eta \frac{\partial}{\partial \eta}\right) W=0
$$

Since the boundary is given by $\xi$ = constant and the boundary condition, (3), is $\mathrm{w}=$ constant (and therefore $\mathrm{W}=$ constant), we seek a solution to $(Q$ ) independent of $\eta$. In this case, $W$ must satisfy the equation

$$
\frac{d^{2} W}{d \xi^{2}} \mp 4 i \xi \frac{d W}{d \xi}=0 .
$$

A solution of (10) is

$$
W(\xi, t w)=R_{ \pm} E^{ \pm}(\xi)
$$

where

$$
E^{ \pm}(\xi)=\int_{\xi}^{\infty} \exp \left[ \pm 2 i \zeta^{2}\right] d \zeta .
$$

We note that $R_{ \pm}$and $\xi$ are functions of $\omega$. It can be shown that when this function is substituted into ( 14 ), $v_{R}$ satisfies the radiation condition. 
Therefore, if $R_{ \pm}$are chosen so as to make $W$ satisfy the boundary condition, (3), $v_{R}$ will be determined. From (3), (7) and (1l), it follows that

(13) $\quad R_{ \pm}=-A(\underline{t w})\left\{E^{ \pm}\left([\lambda r k]^{1 / 2}\right)\right\}^{-1}$

Using (11), (12) and (13) in (2), we obtain

(14) $v_{R}(\underline{x}, \underline{t \omega})=-A(\underline{\omega}) \exp \left[\mp i \lambda k x_{1}\right]\left\{E^{ \pm}\left([\lambda k p]^{1 / 2}\right)\right\}^{-1}\left\{E\left([\lambda k p]^{1 / 2} \cos \frac{\theta}{2}\right)\right\}$. 


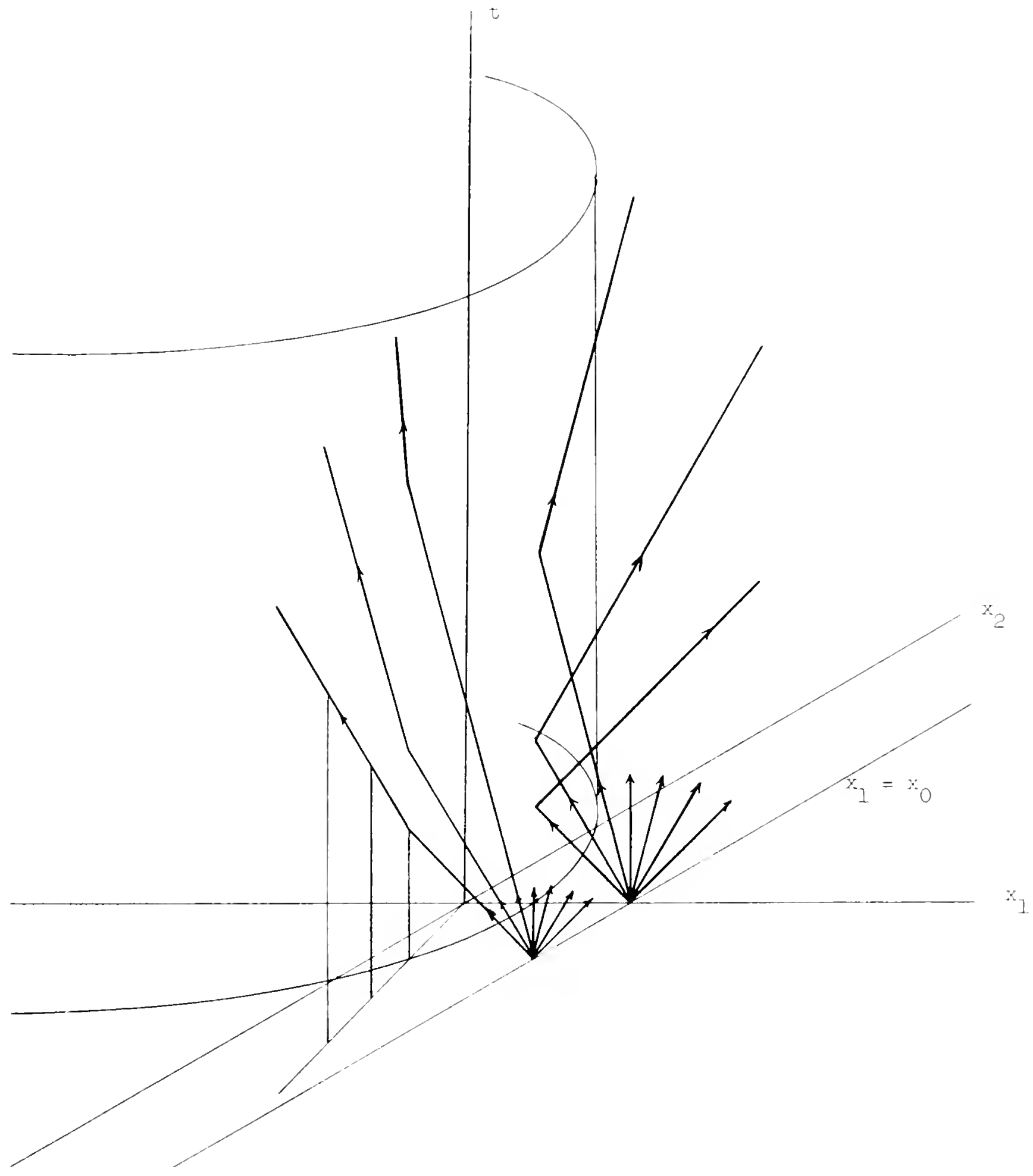

$$
\text { FIE. I }
$$

Ray picture in space-time for the case

$$
\text { of rapidly varying initial data. }
$$


$-35-$

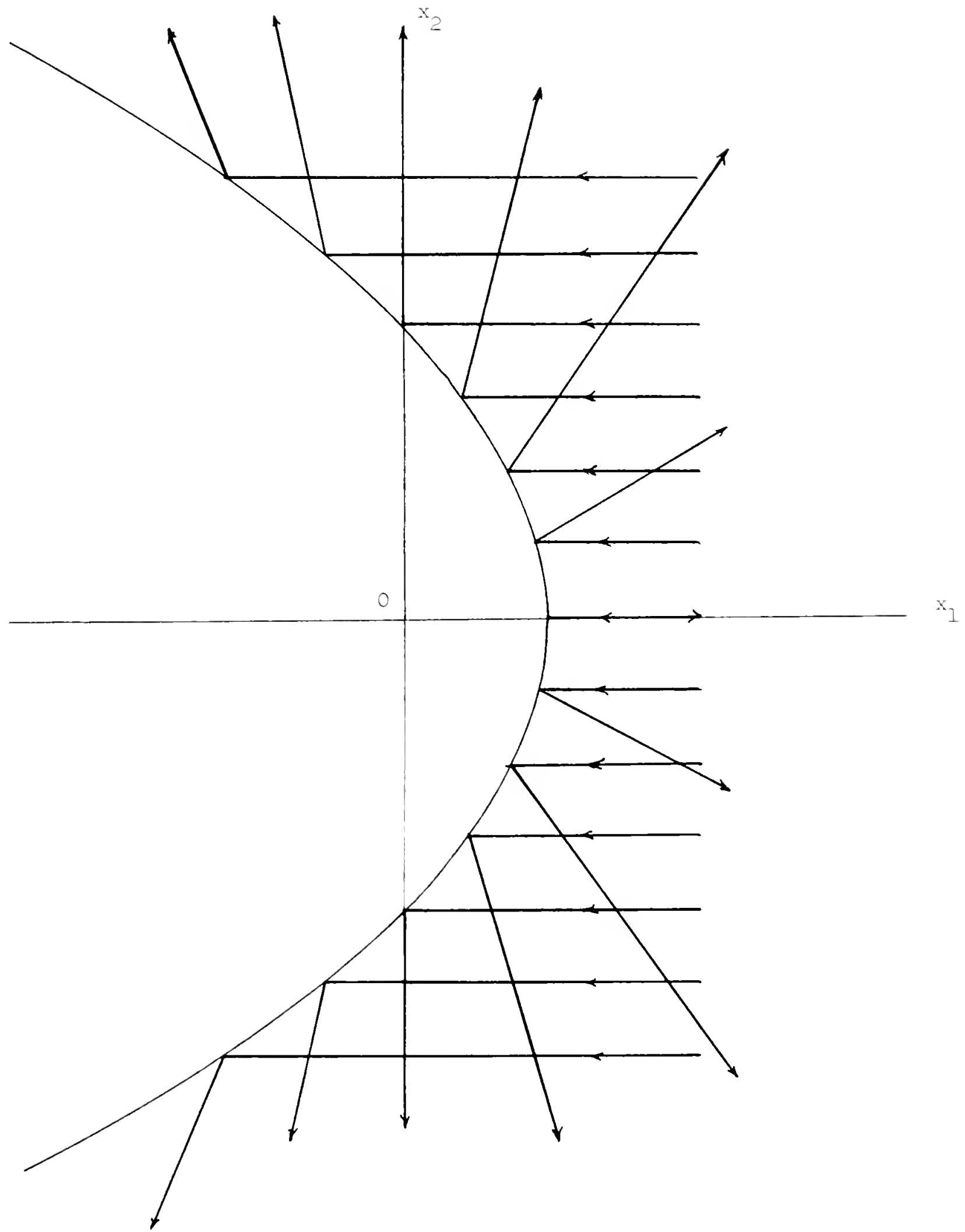

Fig. 2

Projection of rays in space. 


$$
\Pi
$$




\section{References}

[1] Jones, D. The Theory of Electromagnetism, MacMillan (1964).

[2] Keller, J. B. The Geometric Theory of Diffraction, J. Opt. Soc. Amer., 52, 116 (1962).

[3] Lewis, R. M. Asymptotic Methods for the Solution of Dispersive Hyperbolic Equations, Asymptotic Solutions of Differential Equations and their Applications (U. of Wisonsin Symposium Proceedings), 53 (1964).

[4] Lamb, H. On Sommerfeld's Diffraction Problem; and on Reflection by a Parabolic Mirror, Proc. Lona. Math. Soc. (2.) 4, 190 (1905-1906).

[5] Iax, P. E. Asymptotic Solutions of Oscillatory Initial Value Problems, Duke Math. J., 24, 629 (1957).

[6] Bleistein, N. Asymptotic Solution of a Dispersive Hyperbolic Lewis, R. M. Equation with Variable Coefficients, Research Report No. EMp-206, New York University, Courant Institute of Mathematical Sciences, Division of Electromagnetic Research (1965). 

DOCUMENT CONTROL DATA - R\&D

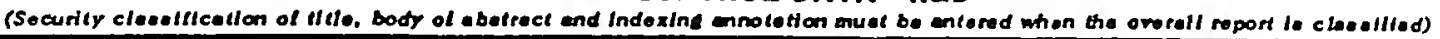
1. ORIGINATIN G ACTIVITY (Comorele author)

New York University

Courant Institute of Mathematical Sciences

Division of Electromagnetic Research

20. REPORT SECURITY CLASSIFICATION

2B. OROUP Unclassified

\section{AEPORT TITLE}

Asymptotic Expansions of Solutions of Initial-Boundary Value Problems for a Dispersive Hyperbolic Equation

\section{RESCAIPTIVE NOTES (TyPe of mport end incluelou doled)}

Scientific Report

3. AUTMOR(S) (Leal namo, Irot name. InIIIal)

Bleistein, Norman, Dr.

Lewis, Robert M., Prof.

\begin{tabular}{|c|c|c|}
\hline $\begin{array}{l}\text { 6. AEPOAT DATE } \\
\text { June, } 1965\end{array}$ & $\begin{array}{l}\text { 70. TOTAL NO. OF PAGES } \\
41\end{array}$ & $\begin{array}{l}\text { 7D. NO. OF MEFS } \\
6\end{array}$ \\
\hline $\begin{array}{l}\text { 8. CONTAACT OR ORANT NO. } \\
\text { AF } 19(628) 4065 \\
\text { B. PROJECT NO } \\
4603,02\end{array}$ & \multicolumn{2}{|c|}{$\begin{array}{l}\text { 90. ORIOINATOR'S REPORT NUMBER(S) } \\
\text { EMP-207 }\end{array}$} \\
\hline $\begin{array}{l}\text { c. DoD element } 62405394 \\
\text { d. DOD subelement } 681000\end{array}$ & \multicolumn{2}{|c|}{ 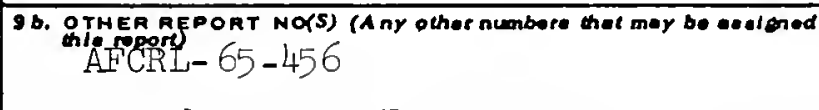 } \\
\hline
\end{tabular}

10.AVALABILITYLIMITATION NOTICES Qualified requestors may obtain copies of this repot from DDC. Other persons or organizations should apply to the Clearinghous for Federal Scientific and Technical. Information (CFSTI), Sills Biulding, 5285 port poral das, springfield Viroinia 22151.

$11 .$. SUPPL EMENTARY NOTES

12. SPONSORING MILITARY ACTIVITY

HQ AFCRL, OAR (CRU)

United States Air Force

L. G. Hanscom Field, Bedford, Mass.

13. ABSTRACT

Initial-boundary value problems for an energy conserving dispersive hyperbolic equation, the Klein-Gordon equation, are considered. This equation exhibits the main feature of dispersion: The speed of propagation depends on frequency. Problems in two space dimensions with a parabolic boundary are discussed.

The primary purpose of this paper is to compare the asymptotic expansion of solutions obtained by a technique we call the ray method with the asymptotic expansion of the exact solution. In the cases considered, the solutions agree.

In addition a numerical comparison is made of the exact and asymptotic solutions for a specified region of space time. 


14.
Key woros
Initial boundary value problems
dispersive hyperbolic equation
asymptotic expansion of solutions
comparison of methods
expansion of exact solution
ray method
two space dimension
parabolic boundary
numerical comparison of exact and
asymptotic solutions

\section{INSTRUCTIONS}

1. ORIGINATING ACTIVITY: Enter the namo and address of the contractor, subcontrsctor, grantee, Departmeot of Defense activity or other organization (corporate author) iseulng the report.

2a. REPORT SECURTY CLASSIFICATION: Enter the over all security clasaification of the report. Indlcate whether "Restricted Dats" la included Marking ls to be in accordance with appropriate security regulstions.

2b. GROUP: Automstic downgrsding is apecifled in DOD Directive 5200.10 and Armed Forcea Induatrial Manual. Enter the group number. A lso, when spplicable, ahow that optional markings have been used for Group 3 and Group 4 as suthcrized.

3. REPORT TITLE: Enter the complete report title in all capital letters. Titles in ali cases should be unclassifled. If a meaningful title csnnot be selected without clsasification, show tille classification in all capitsls In parenthesie immediately following the title.

4. DESCRIPTIVE NOTES: If appropriale, enter the type of report, e.g., interim, progress, summary, snnual, or final.

Give the inclusive dales when a specific reporting period ta covered.

5. AUTHOR(S): Enter the name(s) of author(s) as shown on or in the report. Enter last name, fir st name, middle initiel. If rilitary, show rank and branch of aervice. The name of the principal author is an absolute minimum requirement.

6. REPORT DATE: Enter the date of the report an day, month, year, or month, yesr. If more than ona date appear on the report, use date of publication

7a. TOTAL NUMBER OF PAGES: The total psge count should follow norms! paginstion procedures, $L e_{\text {, }}$, enter tho number of pages contsining Informetion

7b. NUMBER OF REFERENCES Enter the total numbor of referencea cited in the report.

8a. CONTRACT OR GRANT NUMBER: If eppropriste, enter the applicable number of the contrsct or grant under which the report was written

8b, 8c, 8c 8d. PROJECT NUMBER: Enter the approprlate military department identification, such as project number, subproject number, aystem numbers, tssk number, etc.

9a. ORIGINATOR'S REPORT NUMBER(S): Enter the offlcial report number by which the document will be Ident ifled and controlled by the originating ectivity. This number muat be unique to this report.

96. OTHER REPORT NUMBER(S): If the report has been assigned any other report numbers (either by the oridinator or by the sponsor), also enter this number(s).

10. AVAILABILITY/LIMTATION NOTICES: Enter any $1 \mathrm{~lm}$ itations on furtber dlsecmination of the report, other then those imposed by security clasafication, using otenderd statements euch aa:

(1) "Qualifled requesters may obtaln coples of this report irom DDC."

(2) "Forelon announcement and diaseminatlon of this report by DDC Is not authorized."

(3) "U. S. Government agencles may obtsin copies of this report directly from DDC. Other qualified DDC users shall requeat through .10

(4) "U. S. military sgencles may obtain coplea of this report difectly from DDC Other qualified uaers ahall request through

(5) "All distribution of this report is controlled QualIfied DDC usera shall request through

If the report hss been furnished to the Office of Technical Services, Department of Commerce, for sale to the publlc, Indscato this fact and enter the price, if known

11. SUPPLEMENTARY NOTES: Uae for sditional explanatory notes.

12. SPONSORING MILITARY ACTIVITY: Ent er the name of the departmental project office or laboratory sponsaring (pay ing $f o r$ ) the research and development. Include address.

13. ABSTRACT: Enter an absiract giving a brief and factua! summary of the document indicative of the report, even though it may siso appear elsewhere in the body of the technical report. If additionsi apace is required, a continustion aheet shall be attached.

It is highly desirable that the abstract of clasaified reporta be unclassified. Each paragraph of the abstract shall cnd with an indication of the military security ciassification of the ioformation in the psragraph, represented sS (TS), (S), (C), or (U).

There is no limitation on the length of the abstract. However, the auggested length is from 150 to 225 words.

14. KEY WORDS: Key words are technically meaningfil terms or short phrases thet characterize a report and may be used as index entries for cataloging the report. Key words must be aelected 80 that no security clessification ia required. Identeflers, such aa equipment model designation, trade name, military project code name, geographic location, may be used a key worde but will be followed by an indication of technical contoxt. The eseignment of links, rulee, and welghts is optionsl. 


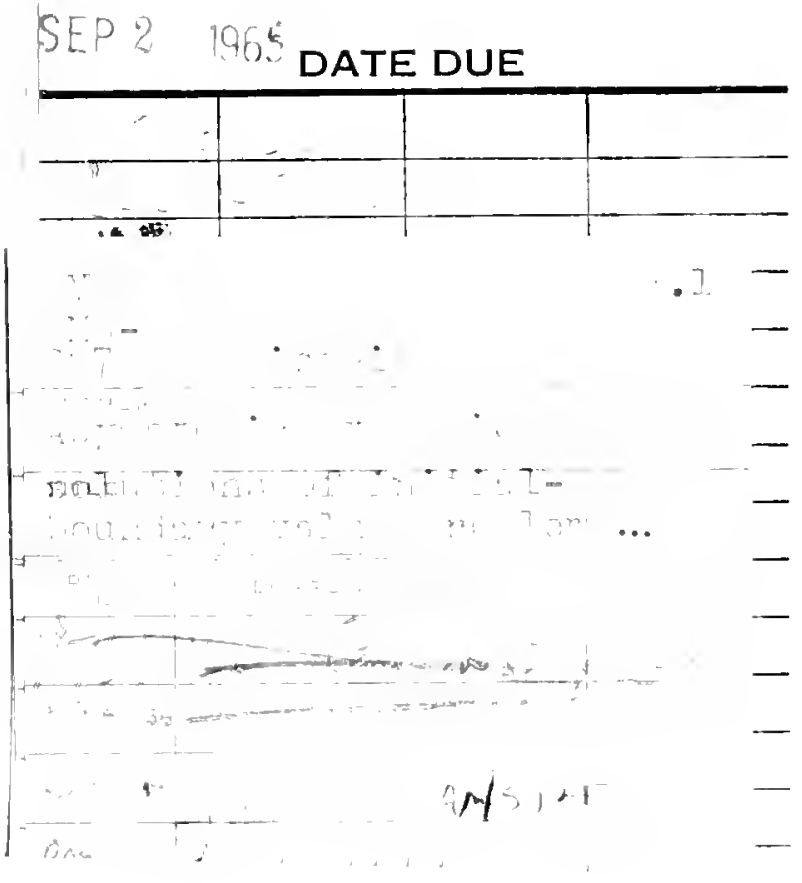

N.Y.U. Courant Institute of Mathematical Sciences 251 Mercer St. New York 12, N. Y. 
